TSUKUBA J. MATH.

Vol. 14 No. 2 )1999), 437-457

\title{
CONSTRUCTION OF INVARIANTS
}

By

\author{
Akihiko GYoJA
}

\section{Introduction.}

Let $G$ be a connected reductive group defined over the complex number field $\boldsymbol{C}, V$ a finite dimensional vector space and $\rho: G \rightarrow G L(V)$ a rational representation of $G$. Such a triplet $(G, \rho, V)$ is called a prehomogeneous vector space if $V$ has an open $G$-orbit, and called irreducible if $\rho$ is an irreducible representation. A complete list of irreducible prehomogeneous vector spaces is given by M. Sato and T. Kimura [12]. The purpose of this paper is to construct explicitly an irreducible relative invariant for every irreducible prehomogeneous vector space. If $(G, \rho, V)$ and $\left(G^{\prime}, \rho^{\prime}, V^{\prime}\right)$ are in the same castling class, then an irreducible relative invariant of $(G, \rho, V)$ can be constructed from that of $\left(G^{\prime}, \rho^{\prime}, V^{\prime}\right)$. (See proposition 18 in $[12$, section 4$]$.) Hence it is enough to consider irreducible reduced prehomogeneous vector spaces. (See [12, section 2] for the generalities concerning the castling transformations.) In the tables I and II of [12, section 7], irreducible relative invariants are given except for the following six cases;

(6) (GL(7), $\left.\Lambda_{3}, V(35)\right)$,

(7) $\left(G L(8), \Lambda_{3}, V(56)\right)$,

(10) $\left(S L(5) \times G L(3), \Lambda_{2} \otimes \Lambda_{1}, V(10) \otimes V(3)\right)$,

(20) (Spin $(10) \times G L(2)$, (half spin) $\left.\otimes \Lambda_{1}, V(16) \otimes V(2)\right)$,

(21) (Spin $(10) \times G L(3)$, (half $\left.\operatorname{spin}) \otimes \Lambda_{1}, V(16) \otimes V(3)\right)$,

(24) $(G L(1) \times \operatorname{Spin}(14)$, (half spin), $V(64))$.

Irreducible relative invariants of (6) and (7) are constructed by $T$. Kimura [8], and that of (20) is constructed by $\mathrm{H}$. Kawahara [7]. (Concerning a construction of an invariant of (7), see the last section of the present paper.) Hence our task is to construct irreducible relative invariants of (10), (21) and (24).

Received July 5, 1989. Revised December 1, 1989. 


\section{Invariants of $S L(5) \times G L(3)$.}

Let $\Lambda^{2} C^{5}$ be the Grassmann tensor product of $\boldsymbol{C}^{5}$ of the second order. If $\left\{e_{1}, \cdots, e_{5}\right\}$ is a basis of $\boldsymbol{C}^{5}$, a general element $x$ of $\Lambda^{2} \boldsymbol{C}^{5}$ is uniquely expressed as

$$
x=\sum_{1 \leq i<j \leq 5} x_{i j} e_{i} \wedge e_{j}
$$

In this section, we reserve the letters $x, y, z, w$ and $u$ for such elements. Their coordinates are written as $x_{i j}, y_{i j}$ etc. and we put $x_{j i}=-x_{i j}$ etc. A general element of the representation space $V=\left(\Lambda^{2} C^{5}\right) \otimes C^{3}$ can be regarded as a triplet $(x, y, z)$ and the action $\rho$ of $G=S L(5) \times G L(3)$ on $V$ is given by

$$
\rho\left(g_{1}, g_{2}\right)(x, y, z)=\left(g_{1} x, g_{1} y, g_{1} z\right) \cdot{ }^{t} g_{2}
$$

for $\left(g_{1}, g_{2}\right) \in G$, where $g_{1} x$ etc. are the natural action of $S L(5)$ on $\Lambda^{2} C^{5}$. Consider the following polynomials;

$$
\begin{aligned}
& f_{1}(x)=x_{23} x_{45}-x_{24} x_{35}+x_{25} x_{34}, \\
& f_{2}(x)=x_{34} x_{51}-x_{35} x_{41}+x_{31} x_{45}, \\
& f_{3}(x)=x_{45} x_{12}-x_{41} x_{52}+x_{42} x_{51}, \\
& f_{4}(x)=x_{51} x_{23}-x_{52} x_{13}+x_{53} x_{12}, \\
& f_{5}(x)=x_{12} x_{34}-x_{18} x_{24}+x_{14} x_{23} .
\end{aligned}
$$

REMARK 1. We introduced these polynomials by a representation theoretic consideration as in [8], so that the property (3) below is satisfied.

Let $D_{y, x}$ be the polarization which transforms a letter $x$ to $y[13]$, In our case

$$
D_{y, x}=\sum_{1 \leq i<j \leq 5} y_{i j} \frac{\partial}{\partial x_{i j}}
$$

Let

$$
g_{i}(x, y)=D_{y, x} f_{i}(x),
$$

and

$$
P(x, y, z, w, u)=\sum_{i, j=1}^{5} g_{i}(x, y) g_{j}(z, w) u_{i, j} .
$$

By the definition of $P$,

$$
P(x, y, z, w, u)=P(y, x, z, w, u)=-P(z, w, x, y, u) .
$$

Hence

$$
P(x, y, x, y, z)=0 \text {. }
$$


LEMMA. The polynomial $P$ is a relative invariant with respect to $G L(5)$. More precisely,

$$
P(g x, g y, g z, g w, g u)=(\operatorname{det} g)^{2} P(x, y, z, w, u)
$$

for $g \in G L(5)$.

PRoOF. Invariance with respect to the scalar action of $G L(1)$ is obvious. By the symmetry, it is enough to show the invariance with respect to the matrix unit $E_{12}$. Note that $-E_{12}$ acts as the polarization which transforms 1 to 2. Hence $-E_{12} f_{2}=-f_{1}$ and $E_{12} f_{i}=0$ for $i \neq 2$. Hence $-E_{12} g_{2}=-g_{1}$ and $E_{12} g_{i}$ $=0$ for $i \neq 2$. Using this fact, we can easily show that $E_{12} P(x, y, z, w, u)=0$.

(4) If at most two kinds of letters appear among $\{x, y, z, w, u\}$, then $P(x, y, z, w, u)=0$, e.g., $P(x, x, x, y, y)=0$ etc.

PROoF. In such a case, $P$ gives a relative invariant of $\left(G L(5), \Lambda_{2} \oplus \Lambda_{2}\right.$, $V(10) \oplus V(10))$ which is a prehomogeneous vector space without relative invariant other than constants $[12 ; \mathrm{p} 94]$. This fact can also be shown by a representation theoretic consideration as in [8].

By (4), $P(z, z, y, y, y)=0$. By the polarization $D_{x, y}$, we get

$$
2 P(z, z, x, y, y)+P(z, z, y, y, x)=0 .
$$

Hence by (1),

$$
2 P(z, z, x, y, y)=P(y, y, z, z, x) .
$$

By (4), $P(y, y, y, x, x)=0$. By the polarization $D_{z, x}$, we get

$$
P(y, y, y, x, z)+P(y, y, y, z, x)=0 \text {. }
$$

By (1) and (7),

$$
P(y, y, y, z, x)=-P(y, y, y, x, z)=P(x, y, y, y, z) .
$$

By multiplying the both sides of (6) and (8),

$$
2 P(y, y, y, z, x) P(z, z, x, y, y)=P(x, y, y, y, z) P(y, y, z, z, x) .
$$

THEOREM 1. Put

$$
\begin{aligned}
F(x, y, z)= & P(x, x, x, y, z) P(y, y, z, z, x)^{2}+P(y, y, y, z, x) P(z, z, x, x, y)^{2} \\
& +P(z, z, z, x, y) P(x, x, y, y, z)^{2} \\
& -P(x, x, y, y, z) P(y, y, z, z, x) P(z, z, x, x, y) \\
& -4 P(x, x, x, y, z) P(y, y, y, z, x) P(z, z, z, x, y) .
\end{aligned}
$$


Then $F$ is an irreducible relative invariant of $\left(S L(5) \times G L(3), \Lambda_{2} \otimes \Lambda_{1}, V(10) \otimes V(3)\right)$ which corresponds to the character

$$
\left(g_{1}, g_{2}\right) \longrightarrow\left(\operatorname{det} g_{2}\right)^{5}, \quad\left(g_{1}, g_{2}\right) \in S L(5) \times G L(3) .
$$

Proof. Since the degree of an irreducible relative invariant is known to be 15 [12, section 7 , Table I (10)], it is enough to prove the relative invariance of $F$. The invariance with respect to $S L(5) \times G L(1)$ is obvious, where $G L(1)$ is the set of scalar matrices in $G L(3)$. Hence it is enough to see the invariance with respect to the actions of the matrix units $E_{i j} \in \operatorname{Lie}(G L(3))$ for $i \neq j$. Since $x, y$ and $z$ appears symmetrically in $F$, it is enough to consider only one of them. The action of $\left\{E_{i j} \mid i \neq j\right\}$ are nothing but the polarizations $D_{y, x}$ etc. Hence it is enough to show that $D_{y, x} F(x, y, z)=0$. By (2) and (4), we have

$$
\begin{aligned}
& D_{y, x} F(x, y, z)=P(x, x, y, y, z) P(y, y, z, z, x)\{P(y, y, z, z, x)-2 P(z, z, x, y, y)\} \\
& \quad+2 P(z, z, x, x, y)\{2 P(y, y, y, z, x) P(z, z, x, y, y)-P(x, y, y, y, z) P(y, y, z, z, x)\} \\
& \quad+4 P(x, x, y, y, z) P(z, z, z, x, y)\{P(x, y, y, y, z)-P(y, y, y, z, x)\}
\end{aligned}
$$

By (6), (9) and (8), the right hand side equals zero.

REMARK 2. Let $G$ be any reductive group and $\rho: G \rightarrow G L(V)$ any rational representation. Let $\left[v_{0}\right] \in V / G$ be a generic point, $v_{0}$ a point in the closed $G$ orbit lying above $\left[v_{0}\right], G_{v_{0}}$ the isotropy subgroup of $G$ at $v_{0}, T$ a maximal torus of $G_{v_{0}}, N$ the normalizer of $T$ in $G, V^{r}=\{v \in V \mid t v=v, t \in T\}, C[V]$ the set of polynomial functions on $V, \phi$ a rational character of $G$ and

$$
\boldsymbol{C}[V]^{G, \phi}=\{f \in \boldsymbol{C}[V] \mid f(g v)=\phi(g) f(v), g \in G\} .
$$

Define $C\left[V^{T}\right]^{N, \phi}$ in the same way. Then we have an isomorphism of Chevalley type

$$
C[V]^{G, \phi} \cong C\left[V^{T}\right]^{N, \phi} .
$$

which is given by the restriction. (See [11; Appendix 2].) For many prehomogeneous vector spaces $(G, \rho, V)$, it is quite easy to give a non-zero element of $C\left[V^{T}\right]^{N, \phi}$. Thus we can describe the restriction of an irreducible relative invariant in $C[V]^{G, \phi}$ to $V^{T}$. In our case, this description gave us enough information to determine the explicit form of $F$ in our theorem.

REMARK 3. In our case $(G, \rho, V)$ has a unique split $Z$-form [3]. For this $\boldsymbol{Z}$-form, $V(\boldsymbol{Z})$ may be identified with the lattice of $V(\boldsymbol{C})$ generated by

$$
\left(e_{i} \wedge e_{j}, 0,0\right),\left(0, e_{i} \wedge e_{j}, 0\right),\left(0,0, e_{i} \wedge e_{j}\right),
$$


where $1 \leqq i<j \leqq 5$. Then $\pm 2^{-5} F(x, y, z)$ are the irreducible relative invariants in $\boldsymbol{Z}[V]$.

In fact, since $g_{i}(x, x)=2 f_{i}(x)$, we can show that $2^{-2} P(x, x, y, y, z)$, $2^{-1} P(y, y, y, z, x)$ etc. belong to $Z[V]$. If we take

$$
\left(e_{1} \wedge e_{2}+e_{3} \wedge e_{4}, e_{2} \wedge e_{3}+e_{4} \wedge e_{5}, e_{1} \wedge e_{3}+e_{2} \wedge e_{5}\right)
$$

as $v_{0}$ in remark 2 , then we can take

$$
\left\{\operatorname{diag}\left(1, t, t^{-1}, t^{2}, t^{-2}\right) \times \operatorname{diag}\left(t^{-1}, 1, t\right) \mid t \in C-\{0\}\right\}
$$

as $T$. Then $C=V^{T}$ is the linear span of the following elements;

$$
\begin{aligned}
& \left(e_{1} \wedge e_{2}, 0,0\right),\left(e_{3} \wedge e_{4}, 0,0\right), \\
& \left(0, e_{2} \wedge e_{3}, 0\right),\left(0, e_{4} \wedge e_{5}, 0\right), \\
& \left(0,0, e_{1} \wedge e_{3}\right),\left(0,0, e_{2} \wedge e_{5}\right) .
\end{aligned}
$$

An easy calculation shows that

$$
\left.2^{-5} F(x, y, z)\right|_{C}=-x_{12}^{3} x_{34}^{2} y_{23} y_{45}^{4} z_{13}^{3} z_{25}^{2} .
$$

Hence $2^{-5} F(x, y, z)$ is irreducible in $Z[V]$. Note that we have also shown that

in our case.

$$
Z[V]^{G, \phi} \cong Z\left[V^{T}\right]^{N, \phi} .
$$

\section{Invariant of $\operatorname{Spin}(10) \times G L(3)$.}

The purpose of this section is to construct an irreducible relative invariant of $\left(\operatorname{Spin}(10) \times G L(3)\right.$, (half spin) $\left.\otimes \Lambda_{1}, V(16) \otimes V(3)\right)$. In this section, we need the theory of spinors. See [12; pp. 110-114] and [1] for the generalities concerning the spinor groups and spinor representations. Here we use the same notations as in [12].

A general element $x$ of the representation space $V(16)$ of the even half spin representation of $\operatorname{Spin}(10)$ can be written uniquely as

$$
x=x_{0}+\sum_{1 \leqq i<j \leqslant 5} x_{i j} e_{i} e_{j}+\sum_{1 \leq i<j<k<l \leqq 5} x_{i j k l} e_{i} e_{j} e_{k} e_{l} .
$$

In this section, we reserve the letters $x, y, z$ and $w$ for such elements. Their coordinates are written as $x_{0}, y_{i j}$ etc., and we put $x_{j i}=-x_{i j}$ and

$$
x_{p(i), p(j), p(k), p(l)}=\operatorname{sign}(p) x_{i j k l}
$$

for any permutation $p$ of $i<j<k<l$. A general element of the representation space $V(16) \otimes V(3)$ can be regarded as a triplet $(x, y, z)$ and the action $\rho=\rho_{1} \otimes \rho_{2}$ 
of $G=\operatorname{Spin}(10) \times G L(3)$ on $V$ is given by

$$
\rho\left(g_{1}, g_{2}\right)(x, y, z)=\left(\rho_{1}\left(g_{1}\right) x, \rho_{1}\left(g_{1}\right) y, \rho_{1}\left(g_{1}\right) z\right) \cdot{ }^{t} \rho_{2}\left(g_{2}\right)
$$

for $\left(g_{1}, g_{2}\right) \in G$, where $\rho_{1}$ is the even half spin representation of $\operatorname{Spin}(10)$ on $V(16)$ and $\rho_{2}$ is the natural representation of $G L(3)$ on $V(3)$.

Consider the following polynomials;

$$
\begin{gathered}
f_{1}(x)=-x_{12} x_{1845}+x_{13} x_{1245}-x_{14} x_{1235}+x_{15} x_{1234}, \\
f_{2}(x)=-x_{23} x_{2451}+x_{24} x_{2351}-x_{25} x_{2841}+x_{21} x_{2345}, \\
f_{3}(x)=-x_{34} x_{3512}+x_{35} x_{3412}-x_{31} x_{3452}+x_{32} x_{3451}, \\
f_{4}(x)=-x_{45} x_{4123}+x_{41} x_{4523}-x_{42} x_{4513}+x_{43} x_{4512}, \\
f_{5}(x)=-x_{51} x_{5234}+x_{52} x_{5134}-x_{53} x_{5124}+x_{54} x_{5123}, \\
f_{6}(x)=x_{0} x_{2345}-x_{23} x_{45}+x_{24} x_{35}-x_{25} x_{34}, \\
f_{7}(x)=x_{0} x_{3451}-x_{34} x_{51}+x_{35} x_{41}-x_{31} x_{45}, \\
f_{8}(x)=x_{0} x_{4512}-x_{45} x_{12}+x_{41} x_{52}-x_{42} x_{51}, \\
f_{9}(x)=x_{0} x_{5123}-x_{51} x_{23}+x_{52} x_{13}-x_{53} x_{12}, \\
f_{10}(x)=x_{0} x_{1234}-x_{12} x_{34}+x_{13} x_{24}-x_{14} x_{23}, \\
g_{i}(x, y)=D_{x y} f_{i}(x), \\
P(x, y, z, w)=\sum_{i=1}^{5}\left(g_{i}(x, y) g_{i+5}(z, w)+g_{i+5}(x, y) g_{i}(z, w)\right),
\end{gathered}
$$

Then by the definition of $P$,

$$
P(x, y, z, w)=P(y, x, z, w)=P(z, w, x, y) .
$$

The polynomials $f_{i}$ are known as spinor invariants [1]. Concerning the properties of the spinor invariants, what is necessary for our purpose is the following fact;

$$
f_{i}\left(\rho_{1}(g) v\right)=\sum_{j=1}^{10} \chi(g)_{i j} f_{j}(v)
$$

for $g \subseteq S \sin (10)$ and $1 \leqq j \leqq 10$. Here $\chi$ denotes the vector representation of Spin (10) ([12]), and $\chi(g)_{i j}$ denote the matrix components. Since the image of $\chi$ is the special orthogonal group which preserves the symmetric bilinear form

$$
\sum_{i=1}^{5}\left(\xi_{i} \eta_{i+5}+\xi_{i+5} \eta_{i}\right)
$$

the polynomial $P$ is a $\operatorname{Spin}(10)$-invariant, i. e., 


$$
P(g x, g y, g z, g w)=P(x, y, z, w)
$$

for $g \equiv \operatorname{Spin}(10)$. Here we wrote $g x$ etc. for $\rho_{1}(g) x$ etc. Of course, (3) can also be shown by a direct calculation as in section 2 . Since $P(x, x, x, x)$ is an (absolute) invariant of the non-regular prehomogeneous vector space (Spin(10), half spin, $V(16)$ ) without relative invariants other than constants $[12$; section 7 , Table III $\left.\left(6^{\prime}\right)\right]$,

$$
P(x, x, x, x)=0 \text {. }
$$

Polarizing (4) by $D_{y x}$, we get

$$
P(x, x, x, y)=0 \text {. }
$$

(Here we used (1).) Polarizing (5) again by $D_{y x}$, we get

$$
P(x, x, y, y)+2 P(x, y, x, y)=0 \text {. }
$$

Polarizing (6) by $D_{z y}$, we get

$$
P(x, x, y, z)+2 P(x, y, x, z)=0 .
$$

THEOREM 2 (H. Kawahara [7]). An irreducible relative invariant of $\left(\operatorname{Spin}(10) \times G L(2)\right.$, (half spin) $\left.\otimes \Lambda_{1}, V(16) \otimes V(2)\right)$ is given by $F_{2}(x, y)=P(x, y, x, y)$.

Proof. It is easy to see that $F_{2}(x, y) \neq 0$. (See remark 4 below.) By (3), the invariance with respect to $\operatorname{Spin}(10) \times G L(1)$ is obvious, where $G L(1)$ is the set of scalar matrices in $G L(2)$. By (1) and (5), we have

$$
D_{x y} F_{2}(x, y)=P(x, x, x, y)+P(x, y, x, x)=0 \text {. }
$$

Since $F_{2}(x, y)=F_{2}(y, x), F_{2}(x, y)$ is a relative invariant with respect to $\operatorname{Spin}(10)$ $\times G L(2)$. Since the degree of an irreducible relative invariant is known to be $4[12$; section 7 , Table I $(20)], F_{2}$ is irreducible.

REMARK 4. In the case treated in theorem $2,(G, \rho, V)$ has a unique split $\boldsymbol{Z}$-form [3]. For this $\boldsymbol{Z}$-form, $V(\boldsymbol{Z})$ may be identified with the lattice of $V(\boldsymbol{C})$ generated by the elements

$$
\begin{aligned}
& (1,0),(0,1), \\
& \left(e_{i} e_{j}, 0\right),\left(0, e_{i} e_{j}\right), \quad(1 \leqq i<j \leqq 5), \\
& \left(e_{i} e_{j} e_{k} e_{l}, 0\right),\left(0, e_{i} e_{j} e_{k} e_{l}\right), \quad(1 \leqq i<j<k<l \leqq 5) .
\end{aligned}
$$

Then $\pm F_{2}(x, y)$ are the irreducible relative invariants in $\boldsymbol{Z}[V]$. In order to prove this, take

$$
\left(1+e_{1} e_{2} e_{3} e_{4}, e_{1} e_{5}+e_{2} e_{3} e_{4} e_{5}\right)
$$


as $v_{0}$ in remark 2 . Then we can take as $T$ the inverse image by ( $\chi \times$ identity) of the set of

$$
\operatorname{diag}\left(1, t_{2}, t_{3}, t_{4}, t_{5}^{2} ; 1, t_{2}^{-1}, t_{3}^{-1}, t_{4}^{-1}, t_{5}^{-2}\right) \times \operatorname{diag}\left(t_{5}, t_{5}^{-1}\right)
$$

where $t_{2}, t_{3}, t_{4}, t_{5} \in C-\{0\}$ and $t_{2} t_{3} t_{4}=1$. Then $C=V^{T}$ is the linear span of the following 4 elements;

$$
(1,0),\left(e_{1} e_{2} e_{3} e_{4}, 0\right),\left(0, e_{1} e_{5}\right),\left(0, e_{2} e_{3} e_{4} e_{5}\right) .
$$

An easy calculation shows that

$$
\left.F_{2}(x, y)\right|_{c}=x_{2} x_{1334} y_{15} y_{2345} \text {. }
$$

Hence $F_{2}$ is irreducible in $\boldsymbol{Z}[V]$. We have also shown that

$$
\boldsymbol{Z}[V]^{G, \phi} \cong \boldsymbol{Z}\left[V^{T}\right]^{N, \phi}
$$

in our case.

THEOREM 3. An irreducible relative invariant of $((\operatorname{Spin}(10) \times G L(3)$, (half spin) $\left.\otimes \Lambda_{1}, V(16) \otimes V(3)\right)$ is given by

$$
\begin{aligned}
F_{3}(x, y, z)= & P(x, x, y, y) P(x, y, z, z)^{2}+P(y, y, z, z) P(y, z, x, x)^{2} \\
& +P(z, z, x, x) P(z, x, y, y)^{2}-P(x, x, y, y) P(y, y, z, z) P(z, z, x, x) \\
& +2 P(x, x, y, z) P(y, y, z, x) P(z, z, x, y) .
\end{aligned}
$$

Proof. It is easy to see that $F_{3}(x, y, z) \neq 0$. (See remark 5 below.) By (3), the invariance with respect to $\operatorname{Spin}(10) \times G L(1)$ is obvious, where $G L(1)$ is the set of scalar matrices of $G L(3)$. Since the degree of an irreducible relative invariant is known to be 12 [12; section 7 , Table I (21)], it is enough to show that $D_{x y} F_{3}(x, y, z)=0$. By (1) and (5), we have

$$
\begin{aligned}
D_{x y} F_{3}(x, y, z)= & 2 P(x, y, z, z) P(x, x, y, z)\{P(x, x, y, z)+2 P(x, y, x, z)\} \\
& +2 P(x, x, z, z) P(x, z, y, y)\{P(x, x, y, z)+2 P(x, y, x, z)\} .
\end{aligned}
$$

Hence by $(7), D_{x y} F_{3}(x, y, z)=0$.

REMARK 5. In the case treated in theorem $3,(G, \rho, V)$ has a unique split $\boldsymbol{Z}$-form [3]. For this $\boldsymbol{Z}$-form, $V(\boldsymbol{Z})$ may be identified with the lattice of $V(\boldsymbol{C})$ generated by

$$
\begin{aligned}
& (1,0,0),(0,1,0),(0,0,1), \\
& \left(e_{i} e_{j}, 0,0\right),\left(0, e_{i} e_{j}, 0\right),\left(0,0, e_{i} e_{j}\right), \quad 1 \leqq i<j \leqq 5, \\
& \left(e_{i} e_{j} e_{k} e_{l}, 0,0\right),\left(0, e_{i} e_{j} e_{k} e_{l}, 0\right),\left(0,0, e_{i} e_{j} e_{k} e_{l}\right), \quad 1 \leqq i<j<k<l \leqq 5 .
\end{aligned}
$$


Then $\pm 2^{-4} F_{3}(x, y, z)$ are the irreducible relative invariants in $Z[V]$. In fact, since $g_{i}(x, x)=2 f_{i}(x)$, we can show that $2^{-2} P(x, x, y, y), 2^{-1} P(x, y, z, z)$ etc. belong to $\boldsymbol{Z}[V]$. Hence $2^{-4} F_{3}(x, y, z) \in \boldsymbol{Z}[V]$. If we take

$$
\left(1+e_{1} e_{2} e_{3} e_{4}, e_{1} e_{5}+e_{2} e_{3} e_{4} e_{5}, e_{1} e_{2}+e_{1} e_{3} e_{4} e_{5}\right)
$$

as $v_{0}$ in remark 2 , then we can take as $T$ the inverse image by ( $\chi \times$ identity) of the set of

$$
\operatorname{diag}\left(1,\left(t_{1} t_{2}\right)^{-1}, t_{1}, t_{2},\left(t_{1} t_{2}\right)^{-2} ; 1, t_{1} t_{2}, t_{1}^{-1}, t_{2}^{-1},\left(t_{1} t_{2}\right)^{2}\right) \times \operatorname{diag}\left(\left(t_{1} t_{2}\right)^{-1}, t_{1} t_{2}, 1\right),
$$

where $t_{1}, t_{2} \in C-\{0\}$. Then $C=V^{T}$ is the linear span of the following 6 elements ;

$$
\begin{aligned}
& (1,0,0),\left(e_{1} e_{2} e_{3} e_{4}, 0,0\right), \\
& \left(0, e_{1} e_{5}, 0\right),\left(0, e_{2} e_{3} e_{4} e_{5}, 0\right), \\
& \left(0,0, e_{1} e_{2}\right),\left(0,0, e_{1} e_{3} e_{4} e_{5}\right) .
\end{aligned}
$$

An easy calculation shows that

$$
\left.2^{-4} F_{3}(x, y, z)\right|_{C}=-x_{0}^{3} x_{1234} y_{15} y_{2345}^{3} z_{12}^{2} z_{1345}^{2} .
$$

Hence $2^{-4} F_{3}(x, y, z)$ is irreducible in $\boldsymbol{Z}[V]$. We have also shown that

$$
\boldsymbol{Z}[V]^{G, \phi} \cong \boldsymbol{Z}\left[V^{T}\right]^{N, \phi}
$$

in our case.

\section{Invariants of $\left(G L(1) \times G L(7), \Lambda_{3} \oplus \Lambda_{1}, V(35) \oplus V(7)\right)$.}

The purpose of this and next sections are to construct an irreducible relative invariant of $(G L(1) \times \operatorname{Spin}(14)$, (odd half spin), $V(64))$, where $G L(1)$ acts on $V(64)$ as scalars. First, we need to construct irreducible relative invariants of $\left(G L(1) \times G L(7), \Lambda_{3} \oplus \Lambda_{1}, V(35) \oplus V(7)\right)$, where $G L(1)$ acts on $V(7)$ as scalars. A construction of the irreducible relative invariants of this prehomogeneous vector space is given by T. Kimura. See $[8 ;$ p. 96 , Table A (14)]. Here we give another construction.

Let $\left\{e_{1}, \cdots, e_{7}\right\}$ be a basis of $V(7)$. Then $\left\{e_{i} \wedge e_{j} \wedge e_{k} \mid 1 \leqq i<j<k \leqq 7\right\}$ is a basis of $V(35)$. We write $e_{i j k}$ for $e_{i} \wedge e_{j} \wedge e_{k}$. A general element of $V=V(35)$ $\oplus V(7)$ can be uniqely expressed as

$$
x=\sum_{1 \leqq i<j<k \leqq 7} x_{i j k} e_{i j k} \oplus \sum_{i=1}^{7} x_{i} e_{i} .
$$

Put $x_{j i k}=-x_{i j k}$ etc. If we take

$$
\left(e_{123}+{ }_{567}+e_{145}+e_{246}+e_{347}\right) \oplus e_{4}
$$


as $v_{0}$ in remark 2 , we can take

$$
\left\{\operatorname{diag}\left(t_{1}, t_{2}, t_{3}, 1, t_{1}^{-1}, t_{2}^{-1}, t_{3}^{-1}\right) \mid t_{1} t_{2} t_{3}=1\right\}
$$

as the maximal torus $T$ of $G_{v_{0}}$, where $G=G L(1) \times G L(7)$. (See remark 2 for the notations.) Then $C=V^{T}$ is the linear span of the following 6 elements ;

$$
e_{123}, e_{567}, e_{145}, e_{246}, e_{347}, e_{4} \text {. }
$$

The relative invariants of $\left(N, V^{T}\right)$ are products of

$$
\begin{gathered}
x_{123}^{2} x_{567}^{2} x_{4}^{2}, \\
x_{123}^{2} x_{567}^{2} x_{145} x_{246} x_{347},
\end{gathered}
$$

and scalars. Let $J_{6}$ and $J_{7}$ be the relative invariants of $(G, V)$ whose restrictions are (4.1) and (4.2) respectively.

THEOREM 4. (1) We have

$$
\begin{aligned}
& J_{6}=\Sigma^{\prime} x_{123}^{2} x_{456}^{2} x_{7}^{2} \\
& -2 \Sigma^{\prime} x_{123}^{2} x_{456} x_{457} x_{6} x_{7} \\
& -2 \Sigma^{\prime} x_{123} x_{124} x_{356} x_{456} x_{7}^{2} \\
& +2 \Sigma^{\prime} x_{123} x_{124} x_{356} x_{457} x_{6} x_{7} \\
& +2 \Sigma^{\prime} x_{123} x_{124} x_{356} x_{567} x_{4} x_{7} \\
& -4 \Sigma^{\prime} x_{123} x_{156} x_{246} x_{345} x_{7}^{2} \\
& -4 \Sigma^{\prime} x_{123} x_{145} x_{246} x_{357} x_{6} x_{7}
\end{aligned}
$$

where $\Sigma^{\prime} x_{123}^{2} x_{456}^{2} x_{7}^{2}$ etc. means the sum of distinct terms among

$$
\left\{x_{p(1), p(2), p(3)}^{2} x_{p(4)}^{2}, p(5), p(6) x_{p(7)}^{2} \mid p \in \widetilde{S}_{7}\right\} .
$$

The relative invariant $J_{6}$ corresponds to the character

$$
\left(g_{1}, g_{2}\right) \longrightarrow g_{1}^{2}\left(\operatorname{det} g_{2}\right)^{2}, \quad\left(g_{1}, g_{2}\right) \leqq G L(1) \times G L(7) .
$$

(2) We have

$$
\begin{aligned}
J_{7} & =\Sigma^{\prime} \pm x_{123} x_{124} x_{135} x_{246} x_{357} x_{467} x_{567} \\
& -\Sigma^{\prime} \pm x_{123}^{2} x_{145} x_{246} x_{357} x_{467} x_{567} \\
& +\Sigma^{\prime} \pm x_{123}^{2} x_{145} x_{246} x_{347} x_{567}^{2} \\
& +\Sigma^{\prime} \pm x_{123} x_{124} x_{135} x_{256} x_{347} x_{467} x_{567} \\
& +\Sigma^{\prime} \pm x_{123} x_{124} x_{135} x_{256} x_{367} x_{457} x_{467}
\end{aligned}
$$




$$
\begin{aligned}
& +\Sigma^{\prime} \pm x_{123} x_{124} x_{156} x_{257} x_{346} x_{357} x_{467} \\
& -2 \Sigma^{\prime} \pm x_{123} x_{124} x_{156} x_{257} x_{345} x_{367} x_{467} \\
& -4 \Sigma^{\prime} \pm x_{128} x_{246} x_{356} x_{257} x_{145} x_{167} x_{347}
\end{aligned}
$$

where $\Sigma^{\prime} \pm x_{123} x_{124 . . .}$ etc. means the sum of distinct terms among

$$
\left\{\operatorname{sign}(p) x_{p(1), p(2), p(3)} x_{p(4), p(5), p(6) \ldots \mid} \mid p \in \widetilde{S}_{7}\right\} .
$$

The relative invariant $J_{7}$ corresponds to the character

$$
\left(g_{1}, g_{2}\right) \longrightarrow\left(\operatorname{det} g_{2}\right)^{3}, \quad\left(g_{1}, g_{2}\right) \in G L(1) \times G L(7) \text {. }
$$

REMARK 6. The above formula for $J_{7}$ is already obtained by J. Igusa [5]. A different formula for $J_{7}$ is given in [2]. (See also [8].)

ProOF OF (1). We write

for the monomial

$$
(a b c, d e f, \cdots, i, j, \cdots)
$$

$$
x_{a b c} x_{d e f} \cdots x_{i} x_{j} \cdots,
$$

and

$$
p(a b c, \cdots)
$$

for

$$
(p(a) p(b) p(c), \cdots),
$$

where $p$ is a permutation. Put

$$
\begin{aligned}
& m_{1}=(123,123,456,456,7,7), \\
& m_{2}=(123,123,456,457,6,7), \\
& m_{3}=(123,124,345,567,6,7), \\
& m_{4}=(123,124,356,456,7,7), \\
& m_{5}=(123,124,356,457,6,7), \\
& m_{6}=(123,124,356,567,4,7), \\
& m_{7}=(123,156,246,345,7,7), \\
& m_{8}=(123,145,246,357,6,7) .
\end{aligned}
$$

By considering the invariance with respect to the maximal torus of $G L(1) \times$ $G L(7)$ and the permutation matrices in $G L(7)$, we can show that $J_{6}$ is of the form 


$$
\sum_{k=1}^{8} a_{k}\left(\Sigma^{\prime} m_{k}\right)
$$

with $a_{1}=1$. Since $(34) m_{3}=-m_{3}, \Sigma^{\prime} m_{3}=0$. So we may suppose that $a_{3}=0$. Let us consider the derivation $D_{i j}(i \neq j)$ such that

$$
D_{i j} x_{k l m}=\delta_{j k} x_{i l m}+\delta_{j l} x_{k i m}+\delta_{j m} x_{k l i} \quad(1 \leqq k<l<m \leqq 7),
$$

and

$$
D_{i j} x_{k}=\delta_{j k} x_{i} \quad(1 \leqq k \leqq 7) .
$$

Since $-D_{i j}$ is nothing but the action of the matrix unit $E_{j i}$, it is enough to determine $a_{k}$ 's so that

$$
D_{i j} \sum_{k} a_{k}\left(\Sigma^{\prime} m_{k}\right)=0
$$

If $(i j)=(76)$

appears only in

$$
(123,123,456,457,7,7)
$$

$$
\begin{aligned}
& D_{76}(123,123,456,456,7,7)=D_{76} m_{1}, \\
& D_{76}(123,123,456,457,6,7)=D_{76} m_{2},
\end{aligned}
$$

Hence $2 a_{1}+a_{2}=0, a_{2}=-2$. If $(i j)=(34)$,

$$
(123,123,356,456,7,7)
$$

appears only in

$$
\begin{aligned}
& D_{34}(123,124,356,456,7,7)=D_{34} m_{4}, \\
& D_{34}(123,123,456,456,7,7)=D_{34} m_{1} .
\end{aligned}
$$

Hence $2 a_{1}+a_{4}=0, a_{4}=-2$. If $(i j)=(34)$,

$(123,123,356,457,6,7)$

appears only in

$$
\begin{aligned}
& D_{34}(123,124,356,457,6,7)=D_{34} m_{5} . \\
& D_{34}(123,123,456,457,6,7)=D_{34} m_{2} .
\end{aligned}
$$

Hence $a_{5}=-a_{2}=2$. If $(i j)=(34)$,

$$
(123,123,356,567,4,7)
$$

appears only in

$$
\begin{aligned}
& D_{34}(123,124,356,567,4,7)=D_{34} m_{6}, \\
& D_{34}(123,123,456,567,4,7)=D_{34}(46) m_{2} .
\end{aligned}
$$

Hence $a_{6}=-a_{2}=2$. If $(i j)=(25)$, 
appears only in

$$
\begin{aligned}
& D_{25}(153,126,246,345,7,7)=-D_{25}(13)(25) m_{4}, \\
& D_{25}(123,156,246,345,7,7)=D_{25} m_{7}, \\
& D_{25}(123,126,546,345,7,7)=-D_{25}(465) m_{4} .
\end{aligned}
$$

Hence $a_{7}=2 a_{4}=-4$. If $(i j)=(34)$.

$$
(123,135,246,357,6,7)
$$

appears only in

$$
\begin{aligned}
& D_{34}(124,135,246,357,6,7)=D_{34}(124653) m_{6}, \\
& D_{34}(123,145,246,357,6,7)=D_{34} m_{8}, \\
& D_{34}(123,135,246,457,6,7)=D_{34}(23)(45) m_{5} .
\end{aligned}
$$

Hence $a_{5}+a_{6}+a_{8}=0, a_{8}=-4$. Thus we have completed the proof of (1).

REMARK 7. Let $P_{i}=\left\{p \in \widetilde{S}_{7} \mid p m_{i}=m_{i}\right\}$. Then

$$
\begin{aligned}
& P_{1}=(\Im(123) \Im(456)) \rtimes\langle(14)(25)(36)\rangle, \\
& P_{2}=\Im(123) \times\langle(45),(67)\rangle, \\
& P_{4}=\langle(12),(56),(15)(26)\rangle \times\langle(34)\rangle, \\
& P_{5}=\langle(12),(34)(67)\rangle, \\
& P_{6}=(\Im(12) \times \Im(56)) \rtimes\langle(15)(26)(47)\rangle, \\
& P_{7}=\langle(26)(35),(12)(45),(23)(56)\rangle \cong \Im_{4}, \\
& P_{8}=\langle(24)(35),(23)(45)(67)\rangle,
\end{aligned}
$$

where an isomorphism $\widetilde{S}_{4} \rightarrow P_{7}$ is given by

$$
(12) \rightarrow(26)(35),(23) \rightarrow(12)(45),(34) \rightarrow(23)(56) .
$$

Hence the number of terms apearing in $\Sigma^{\prime} m_{i}(i=1,2,4,5,6,7,8)$ are $70,210,315$, $1260,630,210$ and 1260 respectively. Let $f^{\vee}=f=J_{6}$. Then $f^{\vee}(\operatorname{grad}) f^{s+1}=b(s) f^{s}$ with a polynomial

$$
b(s)=b_{0}(s+1)\left(s+\frac{5}{2}\right)\left(s+\frac{7}{2}\right)^{2}(s+4)(s+5)
$$

[6]. Since $b(0)=f^{\vee}(\operatorname{grad}) f=2^{5} 5^{2} 7^{2}, b_{0}=2^{6}$.

Proof of (2). We keep the conventions above. Put

$$
m_{1}=(123,124,135,246,357,467,567) \text {, }
$$




$$
\begin{aligned}
& m_{2}=(123,124,134,256,357,467,567), \\
& m_{3}=(123,123,145,246,357,467,567), \\
& m_{4}=(123,123,145,246,347,567,567), \\
& m_{5}=(123,124,135,256,347,467,567), \\
& m_{6}=(123,124,135,256,367,457,467), \\
& m_{7}=(123,124,135,267,367,456,457), \\
& m_{8}=(123,124,156,257,346,357,467), \\
& m_{9}=(123,124,156,257,345,367,467), \\
& m_{10}=(123,246,356,257,145,167,347), \\
& m_{11}=(123,123,123,456,457,467,567), \\
& m_{12}=(123,123,124,345,467,567,567), \\
& m_{13}=(123,123,124,356,457,467,567), \\
& m_{14}=(123,123,145,245,367,467,567), \\
& m_{15}=(123,124,125,345,367,467,567), \\
& m_{16}=(123,124,125,346,357,467,567),
\end{aligned}
$$

By considering the invariance with respect to the maximal torus of $G L(1) \times$ $G L(7)$ and the permutation matrices of $G L(7)$, we can show that $J_{7}$ is of the form

$$
\sum_{k=1}^{16} a_{k}\left(\Sigma^{\prime} \pm m_{k}\right),
$$

with $a_{4}=1$. Since (23)(67) $m_{2}=-m_{2},(45) m_{11}=m_{11},(56) m_{13}=m_{13}$ and (12) $m_{14}=m_{14}$, we have

$$
\Sigma^{\prime} \pm m_{2}=\Sigma^{\prime} \pm m_{11}=\Sigma^{\prime} \pm m_{13}=\Sigma^{\prime} \pm m_{14}=0 \text {. }
$$

So we may suppose that $a_{2}=a_{11}=a_{13}=a_{14}=0$. As in the proof of (1), let us determine the coefficients $a_{k}$ so that

$$
D_{i j} \sum_{k} a_{k}\left(\Sigma^{\prime} \pm m_{k}\right)=0 .
$$

If $(i j)=(34)$,

$$
(123,123,123,345,467,567,567) \text {, }
$$

appears only in

$$
D_{34}(123,123,124,345,467,567,567)=D_{34} m_{12}
$$

Hence $a_{12}=0$. If $(i j)=(34)$, 
$(123,123,125,345,367,467,567)$

appears only in

$$
\begin{aligned}
& D_{34}(123,124,125,345,367,467,567)=D_{34} m_{15}, \\
& D_{34}(123,123,125,345,467,467,567)=-D_{34}(45) m_{12} .
\end{aligned}
$$

Hence $a_{15}=-2 a_{12}=0$. If $(i j)=(34)$,

appears only in

$(123,123,125,346,357,467,567)$

$$
\begin{aligned}
& D_{34}(123,124,125,346,357,467,567)=D_{34} m_{16}, \\
& D_{34}(123,123,125,346,457,467,567)=-D_{34}(45) m_{13} .
\end{aligned}
$$

Hence $a_{16}=-a_{13}=0$. If $(i j)=(54)$,

$$
(123,123,145,246,357,567,567)
$$

appears only in

$$
\begin{aligned}
& D_{54}(123,123,145,246,347,567,567)=D_{54} m_{4}, \\
& D_{54}(123,123,145,246,357,467,567)=D_{54} m_{3},
\end{aligned}
$$

Hence $a_{3}=-a_{4}=-1$. If $(i j)=(34)$,

appears only in

$$
(123,123,135,246,357,467,567)
$$

$$
\begin{aligned}
& D_{34}(123,124,135,246,357,467,567)=D_{34} m_{1}, \\
& D_{34}(123,123,145,246,357,467,567)=D_{34} m_{3}, \\
& D_{34}(123,123,135,246,457,467,567)=-D_{34}(23)(45) m_{13} .
\end{aligned}
$$

Hence $a_{1}+a_{3}-a_{13}=0, a_{1}=1$. If $(i j)=(34)$,

appears only in

$(123,123,135,256,347,467,567)$

$$
\begin{aligned}
& D_{34}(123,124,135,256,347,467,567)=D_{34} m_{5}, \\
& D_{34}(123,123,145,256,347,467,567)=-D_{34}(45) m_{3},
\end{aligned}
$$

Hence $a_{3}+a_{5}=0, a_{5}=1$. If $(i j)=(34)$,

$$
(123,123,135,256,367,457,467)
$$

appears only in

$$
\begin{aligned}
& D_{34}(123,124,135,256,367,457,467)=D_{34} m_{6}, \\
& D_{34}(123,123,145,256,367,457,467)=-D_{34}(12)(456) m_{3}, \\
& D_{34}(123,123,135,256,467,457,467)=D_{34}(23)(4567) m_{12},
\end{aligned}
$$


Hence $a_{6}+a_{3}+2 a_{12}=0, a_{6}=1$. If $(i j)=(34)$,

$(123,123,135,267,367,456,457)$

appears only in

$$
\begin{aligned}
& D_{34}(123,124,135,267,367,456,457)=D_{34} m_{7}, \\
& D_{34}(123,123,145,267,367,456,457)=D_{34}(123)(46)(57) m_{14}, \\
& D_{34}(123,123,135,267,467,456,457)=D_{34}(23)(457) m_{13} .
\end{aligned}
$$

Hence $a_{7}+a_{14}-a_{13}=0, a_{7}=0$. If $(i j)=(34)$,

$(123,123,156,257,346,357,467)$

appears only in

$$
\begin{aligned}
& D_{34}(123,124,156,257,346,357,467)=D_{34} m_{8}, \\
& D_{34}(123,123,156,257,346,457,467)=D_{34}(23)(46) m_{3} .
\end{aligned}
$$

Hence $a_{8}+a_{3}=0, a_{8}=1$. If $(i j)=(34)$,

$$
(123,123,156,257,345,367,467)
$$

appears only in

$$
\begin{aligned}
& D_{34}(123,124,156,257,345,367,467)=D_{34} m_{9}, \\
& D_{34}(123,123,156,257,345,467,467)=-D_{34}(4567) m_{4} .
\end{aligned}
$$

Hence $a_{9}+2 a_{4}=0, a_{9}=-2$. If $(i j)=(34)$,

appears only in

$(123,236,356,257,145,167,347)$

$$
\begin{aligned}
& D_{34}(124,236,356,257,145,167,347)=-D_{34}(24573) m_{9} . \\
& D_{34}(123,246,356,257,145,167,347)=D_{34} m_{10}, \\
& D_{34}(123,236,456,257,145,167,347)=D_{34}(123)(4657) m_{9} .
\end{aligned}
$$

Hence $-a_{9}+a_{10}-a_{9}=0, a_{10}=-4$. Thus we have completed the proof of (2).

REMARK 8. Let $P_{i}=\left\{p \in \Im_{7} \mid p m_{i}=\operatorname{sign}(p) m_{i}\right\}$. Then

$$
\begin{aligned}
& P_{1}=\langle(1357642),(17)(26)(35)\rangle \cong Z_{2} \ltimes Z_{7}, \\
& P_{3}=\langle(23)(45)(67)\rangle \cong Z_{2}, \\
& P_{4}=\langle(12)(56),(23)(67)\rangle \rtimes\langle(17)(26)(35)\rangle \cong \Im_{3} \rtimes Z_{2}, \\
& P_{5}=\langle(23)(45)(67)\rangle \cong Z_{2}, \\
& P_{6}=\langle(17)(26)(34)\rangle \cong Z_{2},
\end{aligned}
$$




$$
\begin{aligned}
& P_{8}=\langle(156)(274)\rangle \cong \boldsymbol{Z}_{3}, \\
& P_{9}=\langle(12)(67),(16)(27)\rangle \rtimes\langle(34)\rangle \cong \boldsymbol{Z}_{2}^{2} \rtimes \boldsymbol{Z}_{2}, \\
& P_{10} \cong S L_{3}\left(\boldsymbol{Z}_{2}\right) .
\end{aligned}
$$

(Note that $S L_{3}\left(\boldsymbol{Z}_{2}\right)$ is of order 168 and is the automorphism group of the finite projective plane over $\boldsymbol{Z}_{2}$.) Hence the numbers of terms appearing in $\Sigma^{\prime} \pm m_{i}$ $(i=1,3,4,5,6,8,9,10)$ are $360,2520,420,2520,2520,1680,630$ and 30 respectively. Let $f^{\vee}=f=J_{7}$. Then $f^{\vee}(\operatorname{grad}) f^{s+1}=b(s) f^{s}$ with a polynomial

$$
b(s)=b_{0}(s+1)(s+2)\left(s+\frac{5}{2}\right)(s+3)\left(s+\frac{7}{2}\right)(s+4)(s+5)
$$

[9]. Since $b(0)=f^{\vee}(\operatorname{grad}) f=2^{5} 35^{2} 7, b_{0}=2^{4}$.

\section{Invariant of $G L(1) \times \operatorname{Spin}(14)$.}

Our purpose here is to construct an irreducible relative invariant $J_{8}$ of the odd half spin representation (Sipn(14), $\rho, V(64))$. Our method of construction is similar to that of J. Igusa [4]. In this section, we use the same notations as in [12].

A general element $x$ of $V(64)$ can be uniquely expressed as

$$
x=\sum_{i} x_{i} e_{i}+\sum_{i<j<k} x_{i j k} e_{i j k}+\sum_{i<j} x_{i j}^{*} e_{i j}^{*}+x_{L} e_{L}
$$

where

$$
\begin{aligned}
& e_{i j k}=e_{i} e_{j} e_{k} \quad \text { etc., } \\
& e_{L}=e_{1234567}, \\
& e_{i j} e_{i j}^{*}=e_{L} .
\end{aligned}
$$

Put $f_{i j}=f_{i} f_{j}$ etc. $x_{j i k}=-x_{i j k}$ etc. and $x_{1 \ldots \hat{i} \ldots \hat{j} \ldots 7}=(-1)^{i+j-1} x_{i j}^{*}$.

LEMMA. In general, put

$$
\begin{gathered}
\left(\prod_{r<s}\left(1+y_{r s} f_{r s}\right)\right)\left(\sum_{i_{0}} x_{i_{0}} e_{i_{0}}+\sum_{i_{0}<i_{1}<i_{2}} x_{i_{0} i_{1} i_{2}} e_{i_{0} i_{1} i_{2}}+\cdots\right) \\
=\sum_{i_{0}} z_{i_{0}} e_{i_{0}}+\sum_{i_{0}<i_{1}<i_{2}} z_{i_{0} i_{1} i_{2}} e_{i_{0} i_{1} i_{2}}+\cdots
\end{gathered}
$$

Then

$$
z_{i_{0} i_{1} \cdots i_{2 q}}=x_{i_{0} i_{1} \cdots i_{2 q}}+\sum_{p>q}(-1)^{p-q} \sum_{i_{2 q+1}<\cdots<i_{2 p}} \operatorname{Pf}\left(y_{i_{r} i_{s}}\right)_{2 q<r, s \leq 2 p} \cdot x_{i_{0} \cdots i_{2 p} p},
$$

where Pf denotes the Pfaffian.

In fact, 


$$
\begin{aligned}
& z_{i_{0} i_{1} \cdots i_{2 q}} e_{i_{0} i_{1} \cdots i_{2 q}} \\
& =\sum_{p \geq q} \frac{1}{2^{p-q}(p-q) !} \sum_{i_{2 q+1} \cdots i_{2} p}\left(y_{i_{2 q+1} i_{2 q+2}} \cdots y_{i_{2 p-1} i_{2 p}}\right)\left(f_{i_{2 q+1} i_{2 q+2}} \cdots f_{i_{2 p-1} i_{2 p}}\right) \\
& \text { - } x_{i_{0} \cdots i_{2 p}} e_{i_{0} \cdots i_{2 p}} \\
& =\sum_{p \geq q} \frac{1}{2^{p-q}(p-q) !} \sum_{i}\left(y_{i_{2 q+1} i_{2 q+2}} \cdots y_{i_{2 p-1} i_{2 p}}\right) x_{i_{0} \cdots i_{2 p}}(-1)^{p-q} e_{i_{0} \cdots i_{2 q}} \\
& \sum_{p \geq q} \sum_{j_{2 q+1}<\cdots<j_{2 p}} \frac{1}{2^{p-q}(p-q) !} \sum_{\left(i_{2 q+1} \cdots i_{2 p}\right.} \sum_{\left(j_{2 q+1} \cdots j_{2 q}\right)}\left(y_{i_{2 q+1} i_{2 q+2}} \cdots y_{i_{2 p-1} i_{2 p}}\right) \\
& \cdot \operatorname{sign}\left(\begin{array}{lll}
j_{2 q+1} & \cdots & j_{2 p} \\
i_{2 q+1} & \cdots & i_{2 p}
\end{array}\right) x_{i_{0} \cdots i_{2 q} j_{2 q+1} \cdots j_{2 p}}(-1)^{p-q} e_{i_{0} \cdots i_{2 q}} \\
& =\sum_{p \geq q} \sum_{i_{2 q+1}<\cdots<i_{2 p}} \operatorname{Pf}\left(y_{i_{r} i_{s}}\right)_{2 q<r, s \leq 2 p} \cdot x_{i_{0} \cdots i_{2 p}}(-1)^{p-q} e_{i_{0} \cdots i_{2 q}} \cdot
\end{aligned}
$$

By the above lemma, we have

$$
\left(\prod_{r<s}\left(1+x_{\bar{L}}^{-1} x_{r s}^{*} f_{r s}\right)\right) x=\sum_{i_{0}} z_{i_{0}} e_{i_{0}}+\sum_{i_{0}<i_{1}<i_{2}} z_{i_{0} i_{1} i_{2}} e_{i_{0} i_{1} i_{2}}+x_{L} e_{L},
$$

where

$$
\begin{aligned}
z_{i_{0}}= & x_{\bar{L}}^{-2}\left\{(-1)^{i_{0}-1} \sum_{i_{1}<\cdots<i_{6}} \operatorname{Pf}\left(x_{i_{r} i_{s}}^{*}\right)_{1 \leq r, s \leq 6}\right. \\
& +\sum_{i_{1}<\cdots<i_{4}} \operatorname{Pf}\left(x_{i_{r} i_{s}}^{*}\right)_{1 \leq r, s \leq 4} \cdot x_{i_{0} i_{1} \cdots i_{4}} \\
& \left.+x_{L_{i_{1}<i_{2}}} x_{i_{1} i_{2}}^{*} x_{i_{0} i_{1} i_{2}}\right\}+x_{i_{0}}
\end{aligned}
$$

and

$$
\begin{aligned}
z_{i_{0} i_{1} i_{2}}= & x_{L}^{-1}\left\{(-1)^{i_{0}+i_{1}+i_{2}} \sum_{i_{3}<\cdots<i_{6}} \operatorname{Pf}\left(x_{i_{r} i_{s}}^{*}\right)_{3 \leq r, s \leq 6}\right. \\
& \left.-\sum_{i_{3}<i_{4}} x_{i_{3} i_{4}}^{*} x_{i_{0} i_{1} \cdots i_{4}}\right\}+x_{i_{0} i_{1} i_{2}} .
\end{aligned}
$$

As is easily seen, every generic element of $\boldsymbol{C}^{7} \oplus \Lambda^{3} C^{7} / S L_{7}(\boldsymbol{C})$ has a representative of the form

$$
w^{\prime} e_{7}+w\left(e_{123}+e_{456}\right)+w^{-1}\left(e_{147}+e_{257}+e_{367}\right),
$$

(cf. [9; Prop. 2.14]). Hence if we put

then

$$
z=\sum z_{i_{0}} e_{i_{0}}+\sum z_{i_{0} i_{1} i_{2}} e_{i_{0} i_{1} i_{2}}+x_{L} e_{L},
$$

$$
\rho(g) z=w^{\prime} e_{7}+w\left(e_{123}+e_{456}\right)+w^{-1}\left(e_{147}+e_{257}+e_{367}\right)+x_{L} e_{L}
$$

with some $w, w^{\prime}$ and $g \in S L(7)(\subset S p i n(14))$. By theorem 4,

and

$$
J_{6}(z)=J_{6}(\rho(g) z)=w^{4} w^{\prime 2},
$$




$$
J_{7}(z)=J_{7}(\rho(g) z)=-w \text {. }
$$

Here we regard $J_{6}$ and $J_{7}$ as polynomial functions on $V(64)$ via the natural projection $V(64) \rightarrow C^{7} \oplus\left(\wedge^{3} C^{7}\right)$. Hence

$$
w=-J_{7}(z), \quad w^{\prime}=\left(J_{6}(z) J_{7}(z)^{-4}\right)^{1 / 2} .
$$

Let $U$ be the linear span of

$$
\left\{e_{7}, e_{123}, e_{456}, e_{147}, e_{257}, e_{367}, e_{L}\right\} \text {. }
$$

Since $J_{8}$ is invariant with respect to the action of $\left\{\Pi_{i=1}^{7}\left(t_{i} e_{i} f_{i}+t_{i}^{-1} f_{i} e_{i}\right) \mid t_{i} \in \boldsymbol{C}-\right.$ $\{0\}\}$ and $\operatorname{deg} J_{8}=8[12$; section 7 , Table $I(24)]$, we can see that $\left.J_{8}\right|_{v}$ is of the form

Since

$$
a x_{7}^{2} x_{123}^{2} x_{456}^{2} x_{L}^{2}+b x_{123}^{2} x_{456}^{2} x_{147} x_{257} x_{367} x_{L} .
$$

$$
\begin{aligned}
& \left(1+2^{-1} f_{14}\right)\left(1+2^{-1} f_{25}\right)\left(1+2^{-1} f_{36}\right)\left(1+e_{14}\right)\left(1+e_{25}\right)\left(1+e_{36}\right) \\
& \quad \cdot\left(e_{7}+e_{123}+e_{456}+e_{1425367}\right) \\
& =e_{123}+e_{456}+2^{-1}\left(e_{147}+e_{257}+e_{367}\right)+2 e_{1425367},
\end{aligned}
$$

we have

$$
\begin{aligned}
& J_{8}\left(e_{7}+e_{123}+e_{456}-e_{L}\right) \\
& =J_{8}\left(e_{123}+e_{456}+2^{-1}\left(e_{147}+e_{257}+e_{367}\right)-2 e_{L}\right) .
\end{aligned}
$$

Hence $a=-b / 4$, and

$$
\left.J_{8}\right|_{U}=x_{7}^{2} x_{123}^{2} x_{456}^{2} x_{L}^{2}-4 x_{123}^{2} x_{456}^{2} x_{147} x_{257} x_{367} x_{L}
$$

up to non-zero scalar multiple. Thus

$$
\begin{aligned}
J_{8}(x) & =J_{8}(z), & & \text { by }(5.1) \\
& =J_{8}\left(w^{\prime} e_{7}+w\left(e_{123}+e_{456}\right)+w^{-1}\left(e_{147}+e_{257}+e_{367}\right)+x_{L} e_{L}\right), & & \text { by }(5.4) \\
& =w^{\prime 2} w^{4} x_{L}^{2}-4 w x_{L}, & & \text { by (5.6) } \\
& =J_{6}(z) x_{L}^{2}+4 J_{7}(z) x_{L}, & & \text { by }(5.5)
\end{aligned}
$$

THEOREM 5. An irreducible relative invariant $J_{8}$ of $(G L(1) \times \operatorname{Spin}(14)$, (odd half spin), $V(64)$ ) is given by

$$
J_{8}(x)=J_{6}(z) x_{L}^{2}+4 J_{7}(z) x_{L}
$$

with

$$
z=\sum z_{i_{0}} e_{i_{0}}+\sum_{i_{0}<i_{1}<i_{2}} z_{i_{0} i_{1} i_{2}} e_{i_{0} i_{1} i_{2}}+x_{L} e_{L}
$$

where $z_{i_{0}}$ and $z_{i_{0} i_{1} i_{2}}$ are given by (5.2) and (5.3). 
REMARK 9. In the case treated in theorem $5,(G, \rho, V)$ has a unique split $\boldsymbol{Z}$-form [3]. For this $\boldsymbol{Z}$-form, $V(\boldsymbol{Z})$ may be identified with the lattice of $V(\boldsymbol{C})$ generated by

$$
e_{i_{0}} e_{i_{1}} \cdots e_{i_{2 k}}, \quad 0 \leqq k \leqq 3,1 \leqq i_{0}<\cdots<i_{2 k} \leqq 7 .
$$

Then $\pm J_{8}(x)$ are the irreducible relative invariants in $Z[V]$. In fact, as is seen from theorem 4, (5.2), (5.3) and theorem 5, $J_{8}(x) \in \boldsymbol{Z}\left[V, x_{\mathbf{L}}^{-1}\right] \cap \boldsymbol{C}[V]=\boldsymbol{Z}[V]$. As is seen from (5.6), $J_{8}$ is irreducible in $Z[V]$. If we take

$$
e_{7}+e_{123}+e_{456}+e_{L}
$$

as $v_{0}$ in remark 2 , then we can take as $T$ the inverse image by $\chi: \operatorname{Spin}(14) \rightarrow$ $S O(14)$ of the set of

$$
\operatorname{diag}\left(t_{1}, t_{2}, t_{3}, t_{4}, t_{5}, t_{6}, 1 ; t_{1}^{-1}, t_{2}^{-1}, t_{3}^{-1}, t_{4}^{-1}, t_{5}^{-1}, t_{6}^{-1}, 1\right),
$$

where $t_{1} t_{2} t_{3}=t_{4} t_{5} t_{6}=1$. Then $C=V^{T}$ is the linear span of the following 4 elements ;

As is seen from (5.6),

$$
e_{7}, e_{123}, e_{456}, e_{L}
$$

$$
\boldsymbol{Z}[V]^{G, \phi} \cong \boldsymbol{Z}\left[V^{T}\right]^{N, \phi}
$$

in our case.

By a direct calculation, we can show that

$$
\left(\operatorname{grad} \log J_{8}\right)\left(v_{0}\right)=2 v_{0} .
$$

As is seen from $(5.6), J_{8}\left(v_{0}\right)=1$. Hence $J_{8}\left(\left(\operatorname{grad} \log J_{8}\right)\left(v_{0}\right)\right) J_{8}\left(v_{0}\right)=2^{8}$, and $J_{8}^{\vee}(\operatorname{grad}) J_{8}^{s+1}=b(s) J_{8}^{s}$ with the polynomial

$$
b(s)=2^{8}(s+1)\left(s+\frac{5}{2}\right)\left(s+\frac{7}{2}\right)(s+4)(s+5)\left(s+\frac{11}{2}\right)\left(s+\frac{13}{2}\right)(s+8),
$$

(cf. [11]).

\section{Invariant of $G L(\S)$.}

In [8; Remark 4.6], a construction of an irreducible relative invariant of $\left(G L(8), \Lambda_{3}, V(56)\right)$ is given. In order to write down this relative invariant explicitly, we need to know the explicit form of polynomials

$$
F_{i \frac{1}{1} \cdots i \frac{q}{m-2}}(x) \quad(q=3, m=3)
$$

appeared in [8; Example (II)]. It would be worth noting that, although the explicit form of these polynomials are not given in [8], they can be constructed immediately as follows: Let $D_{8 i}$ be the polarization $i \rightarrow 8$, i.e., $D_{8, i} x_{\alpha \beta i}=x_{\alpha \beta 8}$ 
$(\alpha, \beta \neq 8,1 \leqq i \leqq 8)$. Then

$$
F_{i_{1}^{1}, i_{1}^{2}, i_{1}^{3}}=D_{8, i_{1}^{1}} D_{8, i_{1}^{2}} D_{8, i_{1}^{3}} f,
$$

where $f$ is an irreducible relative invariant of $\left(G L(7), \Lambda_{3}, V(35)\right)$. In order to see that these polynomials satisfy (4.7) and (4.8) of [8], it is enough to notice that $D_{8 i}$ is nothing but the action of the matrix unit $-E_{i 8}$.

\title{
References
}

[1] C. Chevalley, "The Algebraic Theory of Spinors," Columbia University Press, 1954.

[2] G. B. Gurevich, Sur les trivecteurs dans l'éspace à sept dimensions, Dokl. Akad. Nauk SSSR. III (1934), 567-569.

[3] A. Gyoja, Representations of reductive group schemes, preprint.

[4] J. Igusa, A classification of spinors up to dimension twelve, Amer. J. Math. 92 (1970), 997-1028.

[5] J. Igusa, On the arithmetic of a singular invariant, Amer. J. Math. 110 (1988), 197-233.

[6] S. Kasai, On $b$-functions of regular prehomogeneous vector spaces of simple algebraic groups (in Japanese), Surikaiseki Kenkyusho Kokyuroku 629 (1987), 31-81.

[7] H. Kawahara, Prehomogeneous vector spaces related with the spin group, Master Thesis in Japanese, University of Tokyo (1974).

[8] T. Kimura, Remark on some combinatorial construction of relative invariants, Tsukuba J. Math. 5 (1981), 101-115.

[9] T. Kimura, A classfication of prehomogeneous vector spaces of simple algebraic groups with scalar multiplications, J. Algebra 83 (1983), 72-100.

[10] T. Kimura, The $b$-functions and holonomy diagrams of irreducible regular prehomogeneous vector spaces, Nagoya Math. J. 85 (1982), 1-80.

[11] D. Mumford, "Geometric Invariant Theory (second edition)," Springer.

[12] M. Sato and T. Kimura, A classification of irreducible prehomogeneous vector spaces and their relative invariants, Nagoya Math. J. 65 (1977), 1-155.

[13] H. Weyl, "Classical Groups," Princeton University, 1964.

\author{
Institute of Mathematics \\ Yoshida College, \\ Kyoto University, \\ Kyoto, \\ Japan
}

